\title{
Propiedades psicométricas de la versión española de la Escala Temporal de Satisfacción con la Vida (Temporal Satisfaction With Life Scale, TSLS): Un estudio en mayores que acuden a programas universitarios
}

\author{
Laura Galiana ${ }^{1}$, Melchor Gutiérrez ${ }^{1}$, Patricia Sancho ${ }^{2}$, Amparo \\ Oliver ${ }^{1}$ y José M. Tomás ${ }^{1}$ \\ ${ }^{1}$ Universitat de Valencia (España); ${ }^{2}$ Universidad de Zaragoza (España)
}

\begin{abstract}
Para la operacionalización del bienestar se han desarrollado un gran número de medidas, destacando la Escala de Satisfacción con la Vida (SWLS). Para paliar algunos de sus problemas, se desarrolló la Escala Temporal de Satisfacción con la Vida (TSLS). El objetivo de este estudio es la presentación y primera validación de la TSLS en su versión española, en una muestra de personas mayores que acuden a programas universitarios. La muestra estaba formada por 737 personas mayores. Además de la TSLS, se empleó un indicador de satisfacción general con la vida y la SF-8. Los análisis incluyeron estudio de la validez factorial, estimaciones de la fiabilidad y de validez externa. Los resultados del análisis factorial confirmatorio fueron adecuados: $\chi^{2}(87)=454.593 \quad(p<.001), \mathrm{CFI}=.973$; RMSEA=.085, mostrando la adecuación del modelo de tres dimensiones propuesto. La consistencia interna de las tres dimensiones fue alta: alfas de .83 para la satisfacción con la vida pasada, .81 para la presente y .86 para la futura. También los resultados de validez externa fueron los esperados. La TSLS incorpora el eje temporal en la medida de la satisfacción con la vida, con garantías psicométricas. Sus utilidades se comentan en la discusión.
\end{abstract}

Palabras clave: Satisfacción con la vida; envejecimiento activo; modelos de ecuaciones estructurales.

Psychometric properties of the Spanish version of the Temporal Satisfaction with Life Scale (TSLS): A study on elderly attending to university programs. Several measures have been developed for the operalization of well-being, standing out the Satisfaction With Life Scale (SWLS). To cope with some of its problems the Temporal Satisfaction with Life Scale (TSLS) was developed. The aim of this study is to present and validate the Spanish version of the TSLS, in a sample elderly attending to University programs. The sample was composed of 737 elderly. Together with the TSLS, an indicator of general life satisfaction and the SF-8 Health Scale were used. Analyses included the study of factorial validity, estimations of reliability and external validity. Results of the confirmatory factor analysis were adequate: $\chi^{2}(87)=454.593(p<.001)$, CFI=.973; RMSEA=.085, showing appropriate fit for the three-dimension model posed. Internal consistency of the three dimensions was high: alpha values of .83 for past, .81 for present, and .86 for future life satisfaction. Additionally, results of external validity were as expected. The TSLS addresses the temporal axis in the measurement of life satisfaction, with psychometric guarantees. Its utilities are explained in the discussion.

Keywords: Satisfaction with life; successful aging; structural equation modeling.

Correspondencia: Laura Galiana. Departamento de Metodología de las Ciencias del Comportamiento. Universitat de València. Av. Blasco Ibañez, 21. C.P.:46010. Valencia (España). E-mail: laura.galiana@uv.es 
El interés por la investigación en salud mental y bienestar ha crecido durante las últimas décadas, siendo un tópico de interés tanto en contextos académicos como políticos (Abbott, Ploubidis, Huppert, Kuh, y Croudace, 2010; Burns y Machin, 2009; Dolan y White, 2007; Huppert, Keverne, y Bayliss 2005; Ramírez-Fernández, Ortega-Martínez, y Martos-Montes, 2015; Ryan y Deci, 2001; Seligman, 2002; Snyder y López, 2001).

Tradicionalmente, se han identificado dos partes diferentes del constructo del bienestar subjetivo: una parte afectiva (Diener y Emmons, 1984) y una cognitiva, conocida como satisfacción con la vida (Andrews y Withey, 1976). La satisfacción con la vida es un juicio cognitivo, basado en la comparación que la persona realiza entre su propia vida y unos estándares auto-impuestos, que conllevan a una evaluación global de la vida (Pavot y Diener, 1993).

Un aspecto relevante cuando nos aproximamos a este constructo complejo, es su operacionalización o medida. De facto, a lo largo de la literatura científica se han desarrollado un gran número de medidas de satisfacción con la vida. Estas escalas incluyen desde indicadores simples de satisfacción con la vida (Ye, 2007), pasando por escalas breves como el Índice de Satisfacción con la Vida-Bienestar (Life Satisfaction Index-Well-being, LSI-W; Bigot, 1974), hasta escalas multidimensionales de gran complejidad como la Escala Moral del Centro Geriátrico de Filadelfia (Philadelphia Geriatric Center Morale Scale; Lawton, 1975). Entre todos ellos, sin embargo, destaca la Escala de Satisfacción con la Vida (Satisfaction With Life Scale, SWLS; Diener, Emmons, Larsen, y Griffin, 1985), la más utilizada a lo largo de la literatura. Prueba de ello son el gran número de adaptaciones de la escala realizadas en multitud de idiomas francés (Blais, Vallerand, Pelletier, y Briere, 1989), holandés (Arrindell, Meeuwesen, y Huyse, 1991), checo (Lewis, Shevlin, Smekal, y Dorahy, 1999), español (Atienza, Pons, Balaguer, y García-Merita, 2000), o portugués (Laranjeira, 2009; Sancho, Galiana, Gutiérrez, Francisco, y Tomás, 2014)- y diferentes poblaciones -estudiantes (Diener et al., 1985; Harrington y Loffredo, 2001; Kafka y Kozma, 2002; Lewis, Shevlin, Bunting, y Joseph, 1995; Lucas, Diener, y Suh, 1996; Pavot, Diener, Colvin, y Sandvick, 1991; Pavot, y Diener, 1993; Shevlin, Brunsden, y Miles, 1998; Shevlin y Bunting, 1994), muestras generales y específicas de mayores (Gutiérrez, Tomás, Galiana, Sancho, y Cebrià, 2013; Pavot et al., 1991; Tomás, Sancho, Gutiérrez, y Galiana, 2014), presos (Joy, 1990), miembros de órdenes religiosas (McGarrahan, 1991), adultos jóvenes (Arrindell, Heesink, y Feij, 1999), cuidadores (Vitaliano, Russo, Young, Becker, y Maiuro, 1991), o soldados y enfermeros (Smead, 1991).

La SWLS incluye 5 ítems que miden la satisfacción general con la vida. Es, pues, una evaluación global, aunque el contenido de los ítems incluye tanto afirmaciones referidas a condiciones presentes como pasadas. Dada la problemática que puede conllevar no distinguir entre los cambios producidos a lo largo del tiempo en este 
constructo, Pavot, Diener, y Suh (1998) desarrollaron una medida basada en la SWLS que incluye los mismos 5 ítems, referidos al pasado, al presente y al futuro. Esta escala, conocida como la Escala Temporal de Satisfacción con la Vida (Temporal Satisfaction with Life Scale, TSLS), incluye un eje temporal que puede evitar errores en la medición y además puede resultar muy interesante en la investigación aplicada, en la que distintas condiciones pueden afectar (o ser afectadas por) la satisfacción con la vida pasada, presente y futura (esperada). Además, y dado que los humanos anclamos los sucesos y experiencias al tiempo para darles sentido y continuidad, se espera que la perspectiva temporal influya en nuestras actitudes, comportamientos y objetivos (Sailer et al., 2014).

La TSLS ha sido validada en su presentación original (Pavot et al., 1998), una muestra de estudiantes universitarios canadienses (McIntosh, 2001) y en estudiantes universitarios de Mainland China (Ye, 2007). La primera validación obtuvo evidencia de una estructura de tres factores, satisfacción con la vida pasada, presente y futura, correlacionados entre sí (Pavot et al., 1998). Para conseguir un buen ajuste, tuvieron que añadirse tres correlaciones entre errores. McIntosh (2001), por su parte, encontró un pequeño sesgo de aquiescencia en algunos ítems de la escala. Ye (2007) probó el mismo modelo de tres factores, encontrando un ajuste adecuado tan solo tras algunas modificaciones (covariancias de los errores entre los ítems más similares de las subescalas de satisfacción con la vida pasada, presente y futura).

Llegados a este punto, y dada la ausencia de versiones en español de este instrumento, parece necesario su traducción y adaptación a nuestro contexto. No en balde, la satisfacción con la vida es uno de los constructos de mayor interés en el proceso de envejecimiento (Gutiérrez et al., 2013), tal y como apuntan los vastos esfuerzos realizados en el contexto académico para conocer y explicar la satisfacción con la vida de las personas mayores (Diener, 2000).

Así pues, el objetivo de este estudio, es la presentación y primera validación de la TSLS en su versión española, en una muestra de personas mayores que acuden a programas universitarios.

\section{MÉTODO}

\section{Participantes}

La muestra estuvo formada por 737 personas de 55 años de edad o mayores. La edad media fue de 65.41 años $(D T=6.6)$, oscilando entre un mínimo de 55 y un máximo de 92 años. El 69\% fueron mujeres. En cuanto al nivel de estudios, el $28.4 \%$ contaban con estudios primarios, un $41.9 \%$ con estudios secundarios y un $29.7 \%$ con estudios superiores. Finalmente, y con respecto al estado civil, el $67.5 \%$ estaban casados, un $15.7 \%$ eran viudos, un $10 \%$ solteros y un $6.7 \%$ divorciados. 


\section{Instrumentos}

La encuesta incluía variables socio-demográficas y diversas escalas para evaluar variables de tipo sociológico y psicológico. En concreto, las utilizadas en este trabajo han sido: la Escala Temporal de Satisfacción con la Vida, un indicador general de satisfacción con la vida y la Escala SF-8 de Salud.

La Escala Temporal de Satisfacción con la Vida (TSLS; Pavot et al., 1998) es un instrumento formado por 15 ítems. Fue creada tomando como punto de partida los 5 ítems de la Escala de Satisfacción con la Vida (Diener et al., 1985), que fueron reformulados para evaluar la satisfacción con la vida pasada, presente y futura. Las puntuaciones se recogen en una escala tipo Likert con 5 anclajes, desde 1 (totalmente en desacuerdo) a 5 (totalmente de acuerdo). Se llevó a cabo una traducción back-forward. Dado que las propiedades psicométricas de la escala son el principal objeto de esta investigación, las estimaciones de fiabilidad pueden consultarse en la sección de resultados.

También se utilizó un indicador general de satisfacción con la vida: “Actualmente, ¿cómo de satisfecho está con su vida, considerada globalmente?”. Este indicador se ha utilizado en investigaciones previas con propósitos similares (Ye, 2007). Las respuestas podían variar de 1 (nada) a 5 (mucho).

Finalmente, se empleó la Escala SF-8 de Salud (Ware, Kosinski, Dewey, y Gandek, 2001). Esta escala evalúa la salud relacionada con la calidad de vida, mediante 8 ítems que puntúan en una escala tipo Likert con 5 anclajes de respuesta. La consistencia interna para esta muestra fue de .65 .

\section{Procedimiento}

La investigación tiene un diseño de panel, en el que participaron personas que acudían a los programas de aprendizaje a través del ciclo vital de la Universitat de València (Aulas de la Tercera Edad y Unimajors) durante el curso 2014-2015. En este trabajo, se utilizaron los datos recogidos en la primera ola del diseño.

Se invitó a participar a todos los participantes en el programa. Las encuestas tuvieron lugar en las propias aulas, durante sesiones de aproximadamente 30 minutos de duración. Aunque el procedimiento era de papel y lápiz, se contó en todo momento con la presencia de investigadores y entrevistadores entrenados para solucionar posibles dudas que surgiesen. La tasa de respuesta fue del $77.54 \%$.

\section{Análisis de datos}

La validez factorial de la escala fue evaluada mediante un modelo de ecuaciones estructurales, en concreto, un análisis factorial confirmatorio en el que se puso a prueba la estructura original de los autores (Pavot et al., 1998): tres factores, en los que los ítems de satisfacción con la vida pasada, presente y futura, cargaban 
respectivamente en las dimensiones de satisfacción con la vida pasada, presente y futura; dimensiones correlacionadas.

El ajuste del modelo se evaluó mediante diversos índices o criterios ( $\mathrm{Hu}$ y Bentler, 1999; Tanaka, 1993): 1) el estadístico chi-cuadrado; 2) el Comparative Fit Index (CFI; Bentler, 1990), con valores de .90 o mayores (e idealmente mayores de .95, Hu y Bentler, 1999) indicativos de un buen ajuste; y 3) el Root Mean Squared Error of Approximation (RMSEA; Steiger y Lind, 1980), con valores de .08 o menores como indicativos de un ajuste adecuado. Se utilizó estimación por máxima verosimilitud con correcciones robustas de los errores estándar y correlaciones policóricas, dada la naturaleza ordinal y no normal de los datos. El modelo de ecuaciones estructurales fue estimado en EQS.

Además, se llevaron a cabo estimaciones de consistencia interna. Específicamente, se calculó el alfa de Cronbach, el indicador de fiabilidad más ampliamente utilizado, considerado moderado con valores entre .70 y .79 y alto con valores de .80 o mayores (Cicchetti, 1994; Clarck y Watson, 1995).

Finalmente, la validez criterial y nomológica se estableció mediante correlaciones entre las dimensiones de satisfacción con la vida pasada, presente y futura, con los criterios mencionados: indicador de satisfacción con la vida general y salud percibida.

\section{RESULTADOS}

Los resultados del análisis factorial confirmatorio fueron adecuados: $\chi_{(87)}^{2}=454.593 \quad(p<.001), \mathrm{CFI}=.973 ; \mathrm{RMSEA}=.085$ [intervalo de confianza al $95 \%$ .078-.093]. También los resultados analíticos, con todas las saturaciones factoriales elevadas y estadísticamente significativas (Gráfica 1).

La consistencia interna de las tres dimensiones puede considerarse alta, pues se obtuvieron valores de alfa de Cronbach de .83 para la satisfacción con la vida pasada, .81 para la satisfacción con la vida presente y .86 para la satisfacción con la vida futura.

La validez criterial se estableció correlacionando la satisfacción con la vida pasada, presente y futura con el indicador simple de satisfacción con la vida. Todas las relaciones fueron positivas y estadísticamente significativas. La evidencia de validez nomológica se obtuvo a partir de las correlaciones entre las tres dimensiones de la satisfacción con la vida y la salud percibida, con resultados en la línea de lo encontrado en la literatura. El detalle de los resultados puede consultarse en la tabla 1. 
Gráfica 1. Saturaciones factoriales del modelo de ecuaciones estructurales para la Escala Temporal de Satisfacción con la Vida

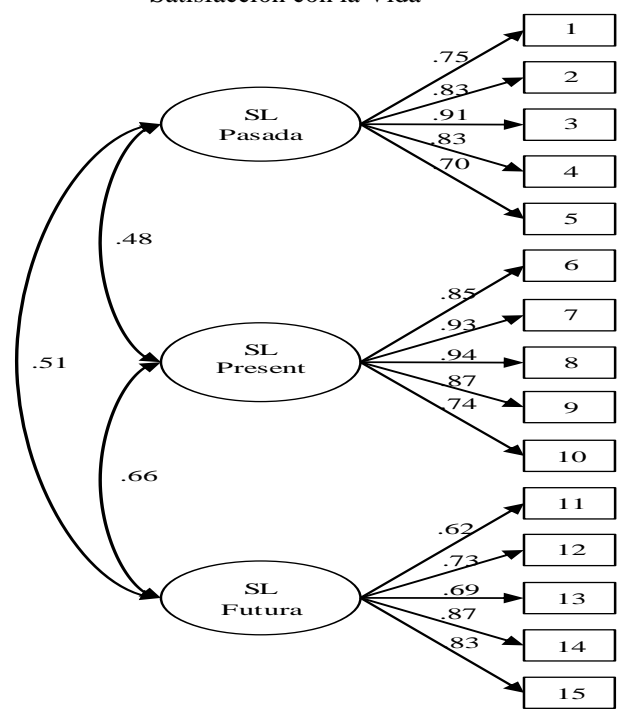

Notas: SL Pasada $=$ Satisfacción con la vida pasada; SL Presente $=$ Satisfacción con la vida presente; SL Futura $=$ Satisfacción con la vida futura. Todas las saturaciones y las correlaciones fueron estadísticamente significativas $(p<0.05)$. Para mayor claridad no se muestran los errores estándar.

Tabla 1. Correlaciones entre las dimensiones de la Escala Temporal de Satisfacción con la Vida, el indicador de satisfacción con la vida global y la salud percibida

\begin{tabular}{lccc}
\hline \multicolumn{1}{c}{ Variables } & SL Pasada & SL Presente & SL Futura \\
\hline Satisfacción con la vida Global & $.499^{* *}$ & $.499^{* *}$ & $.369^{* *}$ \\
\hline Salud percibida & $.209^{* *}$ & $.293^{* *}$ & $.249^{* *}$ \\
\hline
\end{tabular}

Notas: SL Pasada $=$ Satisfacción con la vida pasada; SL Presente $=$ Satisfacción con la vida presente; SL Futura $=$ Satisfacción con la vida futura. ${ }^{* *}=p<0.01$.

\section{DISCUSIÓN}

A pesar de que la Escala de Satisfacción con la Vida (Diener et al., 1985) es una de las más utilizadas para evaluar la satisfacción con la vida, estos mismos autores sugirieron que sería interesante explorar este constructo en términos de las tres franjas temporales de referencia (Pavot et al., 1998). Para ello, desarrollaron la Escala Temporal de Satisfacción con la Vida. Incluir esta perspectiva temporal puede ayudar a desentrañar tanto la medida de "felicidad afectiva", entendida como las subidas y bajadas de las emociones diarias, como la "felicidad evaluativa", la evaluación general de la vida que realiza el individuo (Sachs, 2013). La evaluación de la calidad de vida en mayores y, en concreto, en mayores que acuden a programas universitarios, es un reto de nuestra sociedad actual (Macías, Vives, Orte, y Sánchez, 2014). 
El objetivo de este estudio es validar la TSLS con una doble contribución, una lengua y una población de particular interés: el español, la segunda lengua de comunicación internacional, y las personas mayores, un colectivo clave en la investigación a lo largo del ciclo vital.

La presente validación ofrece resultados de validez factorial, criterial y nomológica, así como de consistencia interna, en una muestra de mayores españoles. En general, los resultados fueron adecuados para una estructura de tres factores, en esta muestra de 737 mayores. De un lado, la validez factorial ofreció evidencia de la existencia de las tres dimensiones propuestas originalmente por los autores, satisfacción pasada, presente y futura. Sin embargo, las correlaciones elevadas y positivas entre éstas dan muestra de cierta estabilidad del constructo a lo largo del tiempo.

Las estimaciones de consistencia interna también fueron apropiadas, con resultados considerados altos (Cicchetti, 1994; Clarck y Watson, 1995). Los alfas de .83, .81 y .86 para la satisfacción con la vida pasada, presente y futura son similares a los encontrados en estudios previos, como por ejemplo en la adaptación alemana de Proyer, Gander, Wyss, y Ruch (2011), con valores de .90, .88 y .88, respectivamente; o en estudiantes suecos (Sailer et al., 2014), en los que se encontraron valores de .86, .93 y .88 , respectivamente. Otros estudios de validación, sin embargo, no ofrecieron estos resultados y, por tanto, no puede realizarse dicha comparación (McIntosh, 2001; Ye, 2007).

Finalmente, la validez criterial y nomológica se evaluaron relacionando la satisfacción con la vida pasada, presente y futura con un indicador de satisfacción con la vida general (Ye, 2007) y la salud percibida (Ware et al., 2001). Los resultados van en la línea de los estudios previos, con correlaciones estadísticamente significativas y positivas entre todas las variables (Gutiérrez et al., 2013; Tomás et al., 2014; Ye, 2007).

Este trabajo, pues, aporta evidencia de cómo la TSLS incorpora el eje temporal en la medida de la satisfacción con la vida, con garantías psicométricas. Su uso puede ser útil en futuras investigaciones centradas en el bienestar y la calidad de vida de las personas mayores, de forma que sus elaboraciones sobre el pasado, el presente y el futuro puedan ser capturadas en los diferentes momentos temporales. Así pues, dado que la satisfacción con la vida no es un constructo completamente estable (Venhooven, 1994), su evaluación mediante tres momentos temporales es prometedora. Además, la medida de la satisfacción con la vida con este enfoque temporal en un cuestionario que no deja de ser breve, puede ser de particular interés para encuestas de mayor tamaño o diseños de investigación longitudinales, en los que la influencia del eje temporal pueda ayudar a esclarecer cambios afectivos y cognitivos.

Destacar, finalmente, que se trata de la primera adaptación y validación de la escala en español, pero también de su primera aplicación, hasta donde conocemos, en 
personas mayores; pues los estudios previos se habían llevado a cabo mayoritariamente en muestras de estudiantes (McIntosh, 2001; Proyer et al., 2011; Ye, 2007).

\section{REFERENCIAS}

Abbott, R.A., Ploubidis, G.B. Huppert, H.A., Kuh, D., y Croudace, T.J. (2010). An evaluation of the precision of measurement of Ryff's Psychological Well-Being Scales in a population sample. Social Indicators Research, 97, 357-373.

Andrews, F.M., y Withey, S.B. (1976). Social indicators of well-being: America's perception of life quality. New York: Plenum.

Arrindell, W.A., Heesink, J., y Feij, J.A. (1999). The satisfaction with life scale (SWLS): Appraisal with 1700 health young adults in the Netherlands. Personality and Individual Differences, 26, 815-826.

Arrindell, W.A., Meeuwesen, L., y Huyse, F.J. (1991). The satisfaction with life scale (SWLS): Psychometric properties in a non-psychiatric medical outpatient's sample. Personality and Individual Differences, 12, 117-123.

Atienza, F.L., Pons, D., Balaguer, I., y García-Merita, M.L. (2000). Propiedades psicométricas de la escala de satisfacción con la vida en adolescentes. Psicothema, 12, 331-336.

Bentler, P.M. (1990). Comparative fit indices in structural models. Psychological Bulletin, 107, 238-246.

Bigot, A. (1974). The relevance of American life satisfaction indices for research on British subjects before and after retirement. Age and Ageing, 2, 113-121.

Blais, M.R., Vallerand, R.J., Pelletier, L.G., y Briere, N.M. (1989). L'Echelle de satisfaction de vie: validation Canadienne-Francaise du "satisfaction with life scale". Canadian Journal of Behavioral Science, 21, 210-223.

Burns, R.A., y Machin, M.A. (2009). Investigating the structural validity of Ryff's Psychological Well-Being Scales across two samples. Social Indicators Research, 93, 359-375.

Cicchetti, D.V. (1994). Guidelines, criteria, and rules of thumb for evaluating normed and standardized assessment instruments in psychology. Psychological Assessment, 6, 284290.

Clarck, L.A., y Watson, D. (1995). Constructing validity: basic issues in objective scale development. Psychological Assessment, 7, 309-319.

Diener, E. (2000). Subjective well-being. American Psychologist, 55, 34-43.

Diener, E., y Emmons, R.A. (1984). The independence of positive and negative affect. Journal of Personality and Social Psychology, 47, 1105-1117.

Diener, E., Emmons, R.A., Larsen, R.J., y Griffin, S. (1985). The satisfaction with life scale. Journal of Personality Assessment, 49, 71-75.

Dolan, P., y White, M. (2007). How can measures of subjective well-being be used to inform public policy? Perspectives on Psychological Science, 2(1), 71-85.

Gutiérrez, M., Tomás, J.M., Galiana, L., Sancho, P., y Cebriá, M.A. (2013). Predicting life satisfaction of the Angolan elderly: A structural model. Aging \& Mental Health, 17(1), 94-101.

Harrington, R., y Loffredo, D.A. (2001). The relationships between life satisfaction, selfconsciousness, and the Myers-Briggs Type Inventory Dimensions. Journal of Psychology, 135, 439-450.

Hu, L., y Bentler, P.M. (1999). Cut-off criteria for fit indexes in covariance structure analysis: Conventional criteria versus new alternatives. Structural Equation Modeling, 6, 1-55. 
Huppert, F.A., Keverne, B., y Bayliss, N. (Eds.). (2005). The science of well-being. Oxford: Oxford University Press.

Joy, R.H. (1990). Path analytic investigation of stress-symptoms relationships: Physical and psychological symptom models. Unpublished doctoral dissertation, University of Illinois at Urbana-Champaign.

Kafka, G.J., y Kozma, A. (2002). The construct validity of Ryff's scales of psychological wellbeing (SPWB) and their relationship to measures of subjective well-being. Social Indicators Research, 57, 171-190.

Laranjeira, C.A. (2009). Preliminary validation study of the Portuguese version of the satisfaction with life scale. Psychology, Health, \& Medicine, 14, 220-226.

Lawton, M.P. (1975). The Philadelphia center morale scale, a revision. Journal of Gerontology, $30,85-89$.

Lewis, C.A, Shevlin, M.E., Smekal, V., y Dorahy, M.J. (1999). Factor structure and reliability of a Czech translation of the satisfaction with life scale among Czech university students. Studia Psychologica, 41, 239-244.

Lewis, C.A., Shevlin, M.E., Bunting, B.P., y Joseph, S. (1995). Confirmatory factor analysis of the satisfaction with life scale: Replication and methodological refinement. Perceptual and Motor Skills, 80, 304-306.

Lucas, R.E., Diener, E., y Suh, E. (1996). Discriminant validity of well-being measures. Journal of Personality Assessment, 71, 3616-3628.

Macías, L., Vives, M., Orte, C., y Sánchez, L. (2014). La importancia de tener buena salud en la autovaloración de la calidad de vida. El ejemplo de los alumnos mayores de las Islas Baleares. European Journal of Investigation in Health, Psychology and Education, 4(2), 141-150.

McGarrahan, J.F. (1991). Family of the origin, antecedents of religious vocation, community experience, and the satisfaction of active and contemplative religious women. Unpublished doctoral dissertation, Temple University.

McIntosh, C.N. (2001). Report on the construct validity of the Temporal Satisfaction with Life Scale. Social Indicators Research, 54, 37-56.

Pavot, W., y Diener, E. (1993). Review of the satisfaction with life scale. Psychological Assessment, 5, 164-172.

Pavot, W., Diener, E., Colvin, C.R., y Sandvick, E. (1991). Further validation of the satisfaction with life scale: Evidence for the cross-method convergence of well-being. Social Indicators Research, 28, 1-20.

Pavot, W., Diener, E., y Suh, E. (1998). The Temporal Satisfaction With Life Scale. Journal of Personality Assessment, 70, 340-354.

Proyer, R. T., Gander, F., Wyss, T., y Ruch, W. (2011). The Relation of Character Strengths to Past, Present, and Future Life Satisfaction among German-Speaking Women. Psychology: Health and Well-Being, 3(3), 370-384.

Ramírez-Fernández, E., Ortega-Martínez, A.R., y Martos-Montes, R. (2015). Las fortalezas en personas mayores como factor que aumenta el bienestar. European Journal of Investigation in Health, Psychology and Education, 5(2), 187-195.

Ryan, R.M., y Deci, E.L. (2001). To be happy or to be self-fulfilled: A review of research on hedonic and eudaimonic well-being. Annual Review of Psychology, 52, 141-166.

Sachs, G. (2013). 25 Ways to see the World Change in 2013. Emerging trends, transformative impacts and how we are helping our clients around the world drive and leverage them. New York: The Goldman Sachs Group, Inc. 
Sailer, U., Rosenberg, P., Nima, A.A., Gamble, A., Gärling, T., Archer, T., y Danilo, G. (2014). A happier and less sinister past, a more hedonistic and less fatalistic present and a more structured future: time perspective and well-being. PeerJ, 2, e303.

Sancho, P., Galiana, L., Gutiérrez, M., Francisco, E., y Tomás, J.M. (2014). Validating the Portuguese Version of the Satisfaction With Life Scale in an Elderly Sample. Social Indicators Research, 115, 457-466.

Seligman, M.E.P. (2002). Authentic Happiness: Using the New Positive Psychology to Realize Your Potential for Lasting Fulfillment. New York: Free Press.

Shevlin, M.E., y Bunting, B.P. (1994). Confirmatory factor analysis of the satisfaction with life scale. Perceptual and Motor Skills, 79, 1316-1318.

Shevlin, M., Brunsden, V., y Miles, J.N.V. (1998). Satisfaction with life scale: Analysis of factorial invariance, mean structures and reliability. Personality and Individual Differences, 25, 911-916.

Smead, V.S. (1991). Measuring well-being is not easy. Paper presented at the Annual Convention of the American Association of Applied and Preventive Psychology.

Snyder, C.R., y López, S.J. (Eds.). (2001). Handbook of Positive Psychology. New York: Oxford University Press.

Steiger, J.H., y Lind, C. (1980). Statistically based tests for the number of common factors. Paper presented at the annual meeting of the Psychometric Society, Iowa City, IA.

Tanaka, J.S. (1993). Multifaceted conceptions of fit in structural equation models. En K.A. Bollen (Ed.), Testing structural equation models (pp. 10-39). Newbury Park, CA: Sage.

Tomás, J.M., Sancho, P., Gutiérrez, M., y Galiana, L. (2014). Predicting Life Satisfaction in the Oldest-Old: A Moderator Effects Study. Social Indicators Research, 117, 601-613.

Venhooven, R. (1994). Is happier a trait? Tests of the theory that a better society does not make people any happier. Social Indicators Research, 32, 101-160.

Vitaliano, P.P., Russo, J., Young, H.M., Becker, J., y Maiuro, R.D. (1991). The screen for caregiver burden. The Gerontologist, 31, 76-83.

Ware, J.E., Kosinski, M., Dewey, J.E., y Gandek, B. (2001). How to Score and Interpret SingleItem Health Status Measures: A Manual for Users of the SF-8 Health Survey. Lincoln RI: QualityMetric Incorporated.

Ye, S. (2007). Validation of the temporal satisfaction with life scale in a sample of Chinese university students. Social Indicators Research, 80, 617-628.

Recibido: 1 de mayo de 2015 Recepción Modificaciones: 15 de septiembre de 2015 Aceptado: 1 de octubre de 2015 